
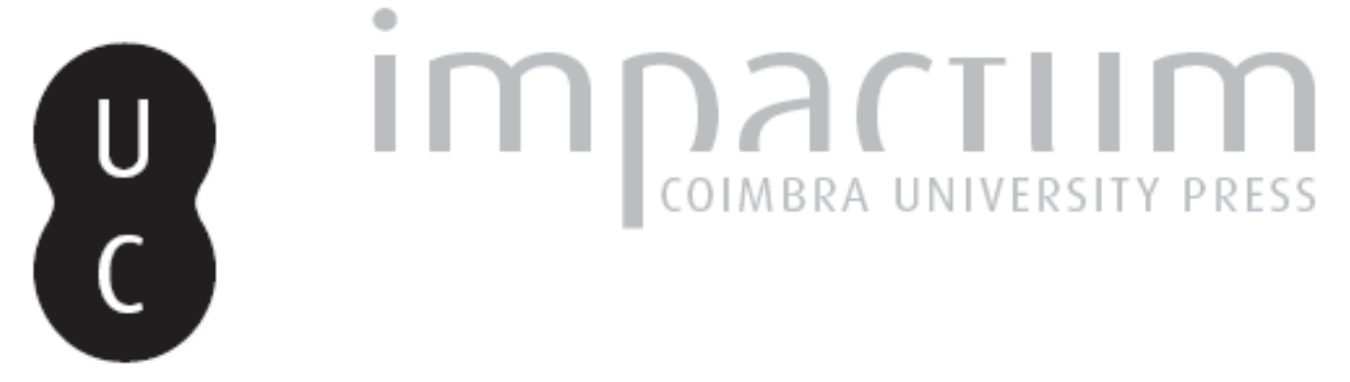

\title{
Triagem diagnóstica da doença pulmonar obstrutiva crónica
}

\author{
Autor(es): $\quad$ Talaia, Mário; Almeida, Catarina
}

Publicado por: $\begin{aligned} & \text { Associação Portuguesa de Riscos, Prevenção e Segurança; Imprensa } \\ & \text { da Universidade de Coimbra }\end{aligned}$

URL

persistente:

URI:http://hdl.handle.net/10316.2/41232

DOI:

DOI:https://doi.org/10.14195/1647-7723_24_12

Accessed : $\quad$ 26-Apr-2023 13:45:47

A navegação consulta e descarregamento dos títulos inseridos nas Bibliotecas Digitais UC Digitalis, UC Pombalina e UC Impactum, pressupõem a aceitação plena e sem reservas dos Termos e Condições de Uso destas Bibliotecas Digitais, disponíveis em https://digitalis.uc.pt/pt-pt/termos.

Conforme exposto nos referidos Termos e Condições de Uso, o descarregamento de títulos de acesso restrito requer uma licença válida de autorização devendo o utilizador aceder ao(s) documento(s) a partir de um endereço de IP da instituição detentora da supramencionada licença.

Ao utilizador é apenas permitido o descarregamento para uso pessoal, pelo que o emprego do(s) título(s) descarregado(s) para outro fim, designadamente comercial, carece de autorização do respetivo autor ou editor da obra.

Na medida em que todas as obras da UC Digitalis se encontram protegidas pelo Código do Direito de Autor e Direitos Conexos e demais legislação aplicável, toda a cópia, parcial ou total, deste documento, nos casos em que é legalmente admitida, deverá conter ou fazer-se acompanhar por este aviso.

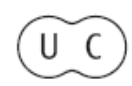




\section{MULTIDISCIPLINARIDADE NA ANÁLISE DAS MANIFESTAÇÕES DE RISCO}

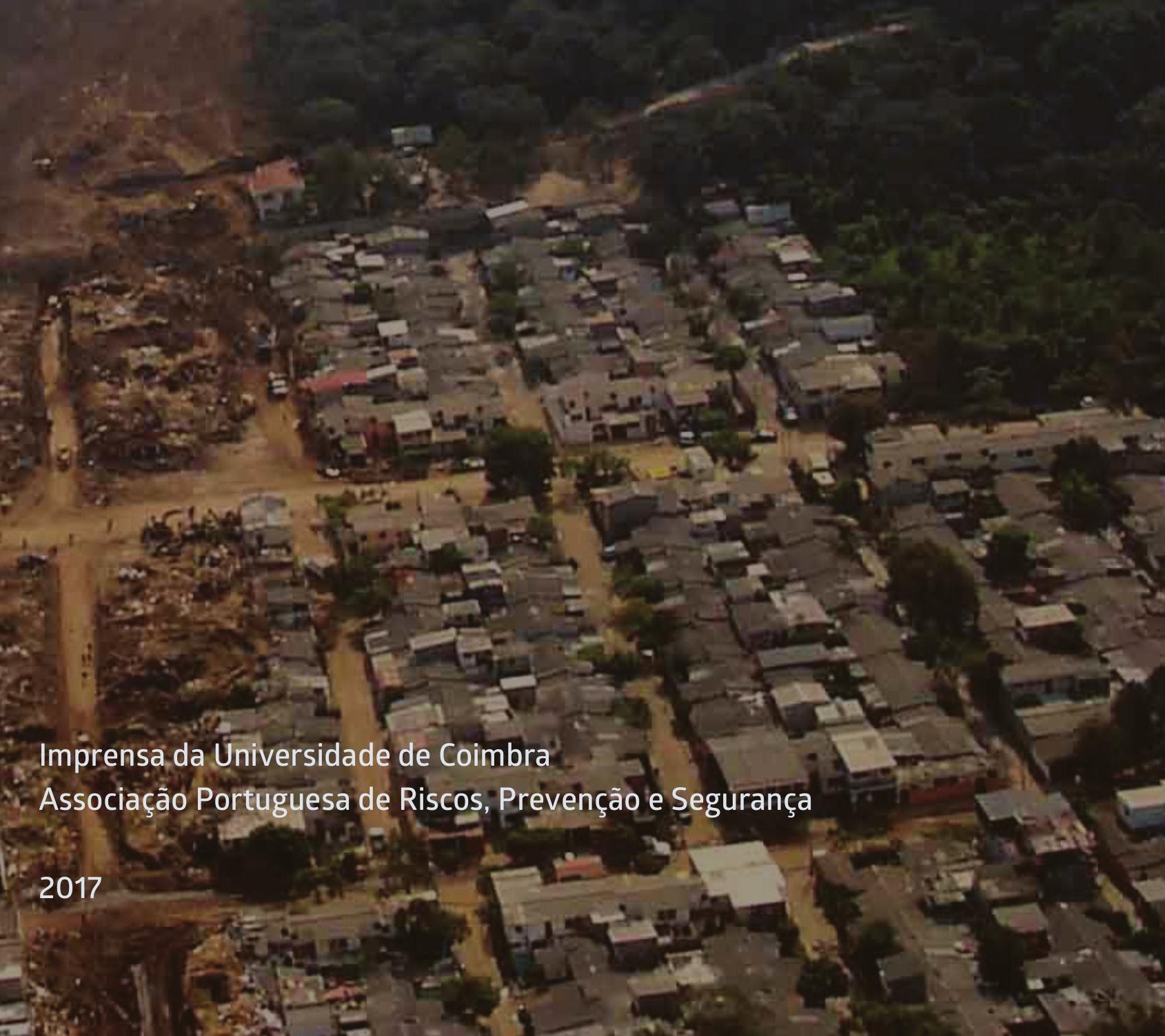




\title{
TRIAGEM DIAGNÓSTICA DA DOENÇA PULMONAR OBSTRUTIVA CRÓNICA*
}

DIAGNOSTIC SCREENING FOR CHRONIC OBSTRUCTIVE PULMONARY DISEASE

Mário Talaia
DFIS-CIDTFF, Universidade de Aveiro
mart@ua.pt

Catarina Almeida

Universidade de Aveiro catarina_259@hotmail.com

\begin{abstract}
RESUMO
A espirometria é o método aceite para o diagnóstico da DPOC (Doença Pulmonar Obstrutiva Crónica), uma doença com elevados custos sociais. Para determinadas profissões, nomeadamente as que envolvem robustez física, o risco da DPOC pode ser avaliado através de uma espirometria. Neste trabalho, a partir de uma colecção de dados, foi avaliado o tipo de patologia: doença ventilatória obstrutiva e/ou doença ventilatória restritiva. Os resultados mostram que o volume expiratório forçado no $4^{\circ}$ segundo deve ser valorizado em substituição da capacidade vital forçada e um novo indicador de triagem foi introduzido a partir do tipo de escoamento.
\end{abstract}

Palavras-chave: Espirometria, robustez física, distúrbios de ventilação, DPOC, índice de Tiffeneau.

\section{ABSTRACT}

Spirometry is the accepted method for COPD diagnosis, a disease with high social costs. For certain professions, especially the ones that involve physical strength, the risk of COPD can be evaluated trough spirometry. In this work, using a data collection, it was evaluated the type of disease (obstructive and/or restrictive). The study shows that the forced expiratory volume in the 4th second should be valued instead of using the forced vital capacity, and a new indicator was introduced, which indicates the type of flow.

Keywords: Spirometry, physical strength, ventilation disorders, COPD, Tiffeneau index.

\section{RESUMEN}

Diagnóstico de detección para la enfermedad pulmonar obstructiva crónica en la aptitud física - La espirometría es el método aceptado como indispensable para el diagnóstico de la EPOC, una enfermedad con altos costos sociales. Para algunas profesiones, especialmente las que implican una aptitud física, el riesgo de EPOC se puede evaluar a través de una espirometría. En este trabajo, con base en el uso de un conjunto de datos, se evaluó el tipo de enfermedad (obstructiva y/o restrictiva). Les resultados muestran que el volumen espiratorio forzado en el $4 \mathrm{~s}$ debe ser valorado en lugar de utilizar la capacidad vital forzada y se ha introducido un nuevo indicador con base en el tipo de flujo.

Palabras clave: Espirometría, fuerza física, trastornos de ventilación, EPOC, índice Tiffeneau.

\section{RESUMÉ}

Diagnostic de maladie pulmonaire obstructive chronique en force physique - La spirométrie est une méthode acceptée pour le diagnostic de la COPD (maladie pulmonaire obstructive chronique), une maladie avec des coûts sociaux élevés. Pour certaines professions, en particulier celles impliquant la force physique, le risque de la COPD peut être évaluée par spirométrie. Dans ce travail à partir d'une collecte de données, a été évalué le type de maladie (obstructive et/ou restrictive). Les résultats montrent que le volume expiratoire forcé en 4 secondes devrait être apprécié à la place de la capacité vitale forcée et une nouvelle fenêtre de sélection a été introduite à partir du type d'écoulement.

Mots-clé: Spirométrie, force physique, des troubles de la ventilation, la MPOC, indice Tiffeneau.

* O texto deste artigo corresponde a uma comunicação apresentada no IX Encontro Nacional de Riscos e II Forum ISCIA, tendo sido submetido em 30-09-2015, sujeito a revisão por pares a 09-01-2016 e aceite para publicação em 20-04-2016.

Este artigo é parte integrante da Revista Territorium, n. ${ }^{\circ} 24,2017,{ }^{\circ}$ RIscos, ISSN: 0872-8941. 
Introdução

A Doença Pulmonar Obstrutiva Crónica ou DPOC é uma patologia crónica, de evolução lenta e progressiva, com grande impacto sobre a função respiratória, que pode estar sujeita a períodos de agudização e complicações graves.

A Organização Mundial de Saúde (OMS) e a Global Initiative for Chronic Obstructive Lung Disease (GOLD) num relatório elaborado pelo National Heart, Lung and Blood Institute (NHLBI), definem a DPOC como: “Uma doença prevenível e tratável com alguns efeitos extrapulmonares importantes que podem contribuir para a gravidade de cada doente. 0 seu componente pulmonar é caracterizado pela limitação do fluxo aéreo, que não é totalmente reversível. A limitação do fluxo aéreo é geralmente progressiva e acompanhada de hiperreactividade brônquica. Esta está associada a uma resposta inflamatória do pulmão à inalação de partículas ou gases nocivos". A DPOC pode ser diagnosticada através do resultado obtido usando uma espirometria ( $F$. C. L. Lundgren et al., 2006; C. Almeida et al., 2012).

A espirometria é um teste fisiológico que mede como um indivíduo inala ou exala volumes de ar, como uma função do tempo. A partir de dados registados numa espirometria, é possível conhecer eventuais distúrbios de ventilação que afectam o normal funcionamento do sistema respiratório (M.R. Miller et al., 2005; L. C. . Silva et al., 2005; P. White, 2003; C. A. S. Pereira, 2002; T. Eaton et al., 1999a; T. Eaton et al., 1999b; J. Terra Filho, 1998).

Para determinadas profissões o despiste de uma avaliação através de uma espirometria é um factor determinante. Por exemplo, este teste faz parte da rotina anual no âmbito da medicina de trabalho para trabalhadores da função pública (programa de vigilância da saúde - serviço de segurança, higiene e saúde no trabalho, de acordo com o artigo $221^{\circ}$ e seguintes e artigo $161^{\circ}$ e seguintes do regime do contrato de trabalho em funções públicas do Anexo I da Lei $n^{\circ}$ 59/2008) e deveria também ser inserida no contexto básico da avaliação clínica de qualquer ser humano. Mesmo nos casos em que, aparentemente, parece não haver distúrbios de ventilação, uma espirometria pode indicar alguns factores de risco e ajudar a prevenir futuros distúrbios respiratórios (P.H. Quanjer et al., 1993; D. E. O’Donnel et al., 1999; G. Fergunson et al., 2000; R.A. Wise e D. P. Tashkin, 2007; M. Arne et al., 2009; A.I. Papaioannou et al., 2009).

A DPOC é uma doença com elevados custos sociais, que tem importância na economia de saúde de um país (A. D. Lopez et al., 2001).

No mundo ocidental constitui a $4^{\text {a }}$ causa de morte nos indivíduos do sexo masculino na idade média de vida, prevendo a Organização Mundial de Saúde (OMS) que em 2020 venha a ocupar a $1^{\text {a }}$ causa de morte e a $3^{\text {a }}$ a nível mundial (C. J. Murray e A. D. Lopes, 1997; D. E. O’Donnell et al., 1999; T. Oga et al., 2003).

É com base nos pressupostos indicados que este trabalho é oportuno por contribuir para uma melhoria na definição de testes para a triagem de diagnóstico da DPOC. Será estudada uma amostra de pacientes que recorreram a testes de espirometria durante um determinado período de tempo e detectar qual o melhor indicador a ser usado sem promover esforço no paciente.

Neste trabalho pretende-se ainda mostrar, nos doentes registados, se o cociente entre o volume expiratório forçado no primeiro segundo $\left(\mathrm{VEF}_{1}\right.$ ou $\left.\mathrm{VEM}_{1}\right)$ e a capacidade vital forçada (CVF) é o único indicador de triagem de diagnóstico de distúrbios ventilatórios e investigar se o volume expiratório forçado aos seis segundos $\left(V_{E F}\right.$ ou $\left.V E M_{6}\right)$ é realmente um substituto viável da CVF, como proposto por alguns autores.

\section{Revisão da literatura e Fundamentação teórica}

Um dos parâmetros mais importantes da espirometria é a Capacidade Vital Forçada (CVF), que é o volume libertado durante uma expiração tão completa e forçada quanto possível, com início na inspiração total. No teste é muito importante registar o Volume Expiratório Forçado libertado no $1^{\circ}$ segundo de uma manobra de CVF, ou seja conhecer o VEM ou VEF . $_{1}$

É difícil realizar uma espirometria em toda a população em risco de desenvolver DPOC, devido ao baixo número de aparelhos portáteis em consultórios médicos assim como à sofisticação dos espirómetros existentes por exigirem a necessidade de técnicos especializados para a realização do exame.

No entanto, a literatura da especialidade mostra a existência de novos índices espirométricos confiáveis, derivados de manobras mais fáceis de executar, para a triagem do diagnóstico da DPOC. Por exemplo, alguns autores (F.L.C. Lundgren et al., 2006; D. Joseph et al., 2004; P.J. Chowienczyk e C.P. Lawson, 1982) sugerem o uso do volume expiratório forçado nos primeiros 6 segundos $\left(\mathrm{VEM}_{6}\right)$ como substituto do CVF na triagem da DPOC. A relação $V E M_{1} / V M_{6}$ parece ser eficaz para o uso na triagem da DPOC e na prática a sua medida é menos desconfortável para o paciente, que não necessita de realizar um esforço expiratório durante muito mais do que 6 segundos e esta vantagem (menos cansativa para o paciente) conduz à menor complexidade de algoritmo (M.P. Swanney et al., 2000; P.L. Enright et al., 2002; M.P. Swanney et al., 2004; J.D. Spahn et al., 2004; J. Vandevoorde et al., 2005; S. Gleson et al., 2006). Actualmente já começam a estar disponíveis valores de referência do VEM (já descritos pela American Thoracic Society e pela European Respiratory Society). 
A prática mostra que quando a relação VEM1/CVF (índice de Tiffeneau) é inferior a $70 \%$ e se valoriza a história clínica e factores de risco compatíveis pode indiciar diagnóstico de DPOC e confirma-se a presença de obstrução no fluxo aéreo que não é totalmente reversível (F.L.C. Lundgren et al., 2006; GOLD, 2007).

A literatura de especialidade mostra que foram desenvolvidos critérios no sentido de avaliar objectivamente a gravidade da doença, conforme se mostra na TABELA I.

A espirometria deve ser considerada um teste simples e de fácil compreensão e obtenção. Conforme mostra C.A.C. Pereira (2002) os valores obtidos devem ser comparados a valores previstos e adequados para a população avaliada.

Para exames espirométricos, é necessário assegurar que os valores funcionais obtidos são exactos. Devem ser usados equipamentos de boa qualidade. Os técnicos devem ter uma boa formação no sentido de levarem os pacientes a realizarem um teste representativo para os vários volumes e fluxos de ar envolvidos. Além disso, é essencial que a tabela de normalidade prevista para o paciente individual seja adequada às suas características, especialmente género, biótipo, idade e etnia.

A maioria dos espirómetros trazem incluídas equações de valores normais previstos no país de origem, são estas referências que são usadas, especialmente a tabela de Knudson (R. J. Knudson et al., 1983; R. J. Knudson et al., 1976).

O espirómetro usado nesta investigação usa como referência a tabela de Knudson para a maioria das variáveis estudadas, como o VEM ao longo do tempo, a CVF, os MEF (Fluxo Expiratório Máximo) a 25\%, 50\% e 75\% da Capacidade Vital e o PEF (Pico de Fluxo Expiratório) para indivíduos com idade igual ou superior a 18 anos.

Segundo M. Jakob (2009) os valores previstos para MEF são determinados a partir da aplicação da expressão,

Homens: $\mathrm{MEF}_{50}=3.79 \mathrm{~L}-0.031 \mathrm{~A}-0.35$

Mulheres: $\mathrm{MEF}_{50}=2.45 \mathrm{~L}-0.025 \mathrm{~A}+1.16$

em que $L$ representa a altura do paciente em metros e $A$ a idade em anos.
Segundo M. Jakob (2009) os valores presentes para VEM, (Volume Expiratório Forçado no primeiro segundo) são determinados a partir da aplicação da expressão,

Homens: $\mathrm{VEM}_{1}=4.301 \mathrm{~L}-0.029 \mathrm{~A}-2.492$

Mulheres: $\mathrm{VEM}_{1}=3.950 \mathrm{~L}-0.025 \mathrm{~A}-2.600$

Os valores de referência local de normalidade para espirometria devem ser revistos periodicamente com a utilização de metodologia adequada, especialmente no que diz respeito à selecção da amostra, precisão dos equipamentos e treino dos técnicos. Algumas diferenças temporais também exigem esta reavaliação, como a maior longevidade e mudanças ambientais e epidemiológicas.

No Brasil já foram obtidos valores de referência para a espirometria e para a população brasileira de raça branca, em 1992. Estes valores foram actualizados em 2006 (C.A.S. Pereira et al., 2007).

O diagnóstico de Doença Ventilatória Obstrutiva (DVO) na presença de CVF normal é simples. A confusão ocorre na presença de DVO quando a CVF é reduzida, devido ao processo obstrutivo ou à restrição associada.

Segundo M.T. Rodrigues et al. (2010), o $\mathrm{MEF}_{50 \%} / 0,5 \mathrm{CVF}$ representa um parâmetro válido no diagnóstico de distúrbios ventilatórios, para pacientes com DVO, DVR (Doença Ventilatória Restritiva) e normais, correlacionando-se positivamente com o VEM/CVF. Um distúrbio restritivo é causado fisiologicamente por redução da Capacidade Pulmonar Total (CPT) (J. L. Clausen, 1989). S.D. Aaron et al. (1999) demonstraram que em $42 \%$ dos casos tidos como restritivos pela espirometria, a CPT não estava reduzida. Estes distúrbios devem ser chamados de inespecíficos (R. E. Hyatt et al., 1997).

M. Green et al. (1974) mostraram que VEM $/$ CVF e $\mathrm{MEF}_{50 \%} / 0,5 \mathrm{CVF}$ registam uma excelente correlação. Se VEM $/$ /CVF já foi consagrada como expressão do fluxo aéreo, é possível afirmar que $\mathrm{MEF}_{50 \%} / 0,5 \mathrm{CVF}$ tem a sua aplicabilidade, sendo uma contribuição no diagnóstico precoce dos distúrbios ventilatórios.

O VEM $M_{t}$ é o volume máximo exalado no tempo t de uma expiração forçada a partir de uma posição de inspiração total. Tem havido um aumento considerável da utilização 
do VEM 6 como um substituto de confiança para a CVF e pode ser utilizado para determinar $V_{E} M_{1} / V M_{6}$. A utilização do VEM tem vantagem face a CVF por não necessitar de tanto esforço físico por parte do paciente e providenciar o mesmo diagnóstico.

\section{Metodologia}

O presente estudo teve por base a análise de espirometrias realizadas no Serviço de Pneumologia de Hospital da região centro de Portugal, no período entre Outubro de 2012 e Janeiro de 2013. Todos os testes foram realizados dentro da rotina do atendimento de pacientes que procuraram a unidade ou que foram encaminhados pelos seus médicos para a obtenção de um diagnóstico. Nenhum teste foi excluído. No que se refere às principais características da investigação quantitativa esta obedeceu a um plano preestabelecido, com o intuito de enumerar ou medir eventos; utilizou a teoria para desenvolver as hipóteses e as variáveis da pesquisa; examinou as relações entre as variáveis por métodos experimentais ou semiexperimentais, controlados com rigor; e empregou para a análise dos dados indicadores estatísticos (N. K., Denzin e Y. S. Lincoln, 2005).

É sabido que toda a investigação é feita numa população e, no caso de a população ser composta por um número relativamente elevado de pessoas será praticamente impossível utilizá-la, por razões de tempo e de custos, e porque não é na realidade imprescindível examinar cada unidade da população. Em vez de realizar esta extenuante tarefa, neste trabalho procede-se à extração de uma amostra dessa população, ou seja, um conjunto de unidades, numa porção do total, que represente a conduta da população no seu conjunto (L. Pardal e E. S. L. Lopes, 2011). Assim, a população é o conjunto de todos os indivíduos que tem uma ou mais características comuns, e que se encontram num espaço ou território conhecido. No entanto, nem todas as amostras são úteis para desenvolver um trabalho de investigação. 0 que se procura é construir uma amostra em que, observando uma porção relativamente reduzida de unidades, se obtenham conclusões semelhantes às que se chegaria se se estudasse o total da população. Quando uma amostra cumpre esta condição, ou seja, quando reflete nas suas unidades o que ocorre na população é chamada de amostra representativa. Todavia, não se pode saber nunca à priori se a amostra obtida é, efetivamente, ou não representativa, pois para se ter a certeza absoluta seria necessário investigar toda a população e, assim, comparar ambos os resultados.

Neste trabalho a amostra é não-probabilística uma vez que segundo R. Huot (2002) os vários elementos da população não possuem a mesma probabilidade de fazer parte da amostra. É um método de carácter pragmático ou intuitivo e largamente utilizado, pois possibilita um estudo mais rápido e menos dispendioso. Um claro inconveniente deste método é o facto da inclusão de um elemento da população na amostra ser determinada por um critério subjetivo, normalmente uma opinião pessoal. Um outro inconveniente é que existem elementos da população que não têm possibilidade de ser escolhidos. As amostras não probabilísticas mais utilizadas são as amostras por quotas, por redes ou as intencionais (L. Cohen et al., 2010). Como já foi referido, neste trabalho ir-se-á utilizar uma amostra não probabilística por conveniência, também chamada acidental, por ser obtida sem nenhum plano preconcebido, resultando de exames realizados num determinado período de tempo.

As espirometrias foram realizadas com o espirómetro MasterScreen Body (CareFusion Corporation, 2012). Este aparelho, graças ao seu conceito modular, é uma estação prática orientada para testes de função pulmonar, para determinação de volumes pulmonares estáticos e dinâmicos, utilizando o método clássico de He-reinalação e a capacidade de difusão via método Sopro Único. As unidades MasterScreen obedecem aos critérios dos standards relevantes, especialmente a ERS (Sociedade Respiratória Europeia) e os muito rigorosos critérios da ATS (American Thoracic Society). A excelente segurança para o paciente é enfatizada, indo de encontro aos standards da SEV (Swiss Safety Standars). O MasterScreenBody é usado para medidas e recolha de dados de parâmetros de função pulmonar. O sistema efectua testes de função pulmonar dependentes da cooperação do paciente, que incluem espirometria/fluxo-volume/medidas de resistência, medidas de difusão do pulmão e medidas de pletismografia corporal. $\mathrm{O}$ aparelho providencia dados/informação e ajuda de suporte para o diagnóstico. 0 equipamento pode ser utilizado em pacientes a partir dos 4 anos de idade, desde que eles consigam cooperar para a realização do teste de diagnóstico. Os testes realizados e apresentados neste trabalho foram obtidos sem recurso a broncodilatadores.

A fig. 1 mostra o equipamento usado e uma fase de um diagnóstico.

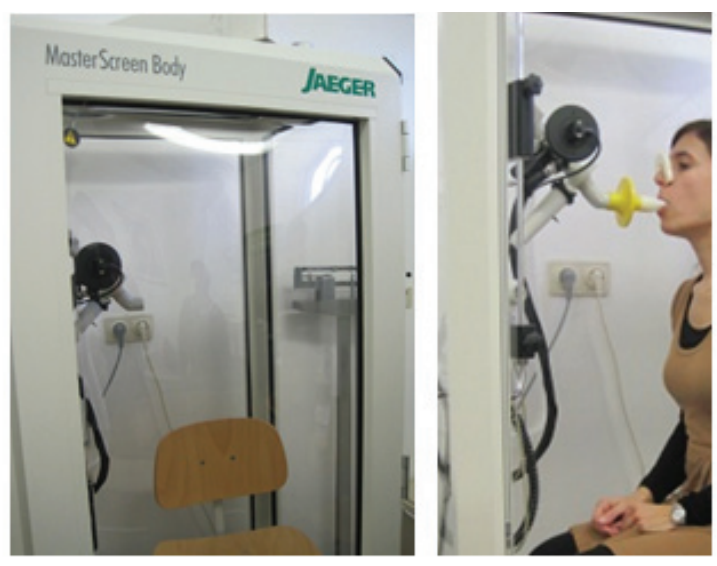

Fig. 1 - Equipamento usado e diagnóstico em curso.

Fig. 1 - Used equipment and diagnostics in progress. 
Cada gráfico obtido em papel foi analisado em termos da necessidade de dados a estudar. Cada exame tem informação do género do paciente, idade, massa corporal e altura. Os dados são registados em computador.

O algoritmo desenvolvido e usado pelo computador escolhe o "Best", ou seja, o melhor dos testes realizados em termos de performance por considerar que foi aquele em que o paciente colaborou melhor. 0 exame considerado "Best" é indicado no gráfico com a linha de cor verde (as outras linhas têm uma cruz de eliminação). No gráfico, a ordenada indica volume (L) e a abcissa tempo (s).

Os resultados das espirometrias foram registados em gráficos volume - tempo. Para cada exame foram determinados os valores de VEM, em que o índice $i$ indica o tempo na linha traçada durante o exame. A linha que começa no instante $\mathrm{t}=0 \mathrm{~s}$ e $\mathrm{V}=\mathrm{OL}$ (como referência de ar a sair) e que passa pelos pontos i é a linha volume - tempo.

Os valores previstos esperados para cada paciente também foram calculados utilizando expressões usadas para homens e mulheres, expressões (1), (2), (3) e (4).

Determinou-se os valores para o índice de Tiffeneau, quer previsto quer real. Para cada exame e tendo por base a identificação de cada paciente foi criada uma base de dados.

Também a partir da linha traçada durante o exame foi possível determinar o Fluxo Expiratório Máximo ou Fluxo Expiratório Forçado a $50 \%$ da Capacidade Vital $\left(\mathrm{MEF}_{50 \%}\right.$ ou $\mathrm{FEF}_{50 \%}$ ) que é independente do esforço, mas dependente em grande parte do volume pulmonar e tamanho das vias aéreas, embora estas duas variáveis não estejam fortemente relacionadas. Neste trabalho o MEF50\% foi determinado a partir da tangente no ponto da curva que regista $50 \%$ da CVF.

A realização de cada exame seguiu os seguintes passos: o técnico obedeceu aos critérios técnicos do equipamento e seguiu o procedimento adaptado; o técnico mostrou que é importante o paciente repousar cerca de 5 minutos antes do exame; o técnico registou os dados do paciente no livro de registo e no programa de software do computador do espirómetro; o técnico explicou o exame ao paciente; o paciente colocou o tubo de respiração na boca e houve certeza de que os lábios estavam selados à volta do tubo e que a língua não estava a tapá-lo; o técnico colocou ao paciente o clipe ou uma oclusão manual nas narinas e pediu ao paciente para respirar normalmente; o técnico pediu ao paciente que enchesse o peito de ar de uma só vez e ao máximo e que depois soprasse com força no início e que prolonga-se o sopro. 0 exame termina. 0 técnico pediu ao paciente para voltar a encher o peito de ar e o exame é repetido mais duas vezes. O programa desenvolvido para o registo de dados escolhe o melhor (Best) dos três exames. No final, o técnico gravou os dados em ficheiro e fez uma impressão do exame. Podem ser colocadas notas junto do exame, como, por exemplo, se o paciente colaborou.

\section{Resultados}

Foram considerados 84 exames em que 48 pertencem ao sexo masculino e 36 ao sexo feminino, como se mostra na TABELA II. No geral o tratamento estatístico da amostra para os dois sexos mostra que a média das idades é 57.1 anos com um desvio padrão de 14.5 anos. A média da altura é $164.9 \mathrm{~cm}$ com um desvio padrão de $9.1 \mathrm{~cm} \mathrm{e}$ uma massa corporal de $79.7 \mathrm{~kg}$ com um desvio padrão de $16.6 \mathrm{~kg}$.

Com base no ficheiro de dados criado, determinou-se os valores de VEM para cada curva de exame e construiu-se a TABELA III. Nesta tabela são indicados para cada VEM 0 número de casos registados e a percentagem para cada resultado obtido.

A observação da TABELA III mostra que apenas 53.6\% dos pacientes atingiram $6 \mathrm{~s}$ para VEM e apenas $9.5 \%$ o tempo de $8 \mathrm{~s}$.

TABELA II - Amostra considerada (valor médio e desvio padrão).

$T_{A B L E}$ II - Sample considered (mean value and standard deviation).

\begin{tabular}{|c|c|c|c|c|}
\hline Número & Género & Idade $(\mathrm{anos})$ & Altura $(\mathrm{cm})$ & Massa $(\mathrm{kg})$ \\
\hline 84 & $\mathrm{MF}$ & $57.1 \pm 14.5$ & $164.9 \pm 9.1$ & $79.7 \pm 16.6$ \\
\hline 48 & $\mathrm{M}$ & $57.7 \pm 13.6$ & $170.0 \pm 7.5$ & $83.7 \pm 17.4$ \\
\hline 36 & $\mathrm{~F}$ & $56.3 \pm 15.8$ & $158.1 \pm 6.1$ & $74.4 \pm 13.9$ \\
\hline
\end{tabular}

TABELA III - VEM em número e em percentagem em face da amostra

TaBLE III - FEVi in number and percentage in face of the sample

\begin{tabular}{|c|c|c|c|c|c|}
\hline VEM $_{1,2,3}$ & VEM $_{4}$ & VEM $_{5}$ & VEM $_{6}$ & VEM $_{7}$ & VEM $_{8}$ \\
\hline 84 & 77 & 62 & 45 & 31 \\
\hline $100.0 \%$ & $91.7 \%$ & $73.8 \%$ & $53.6 \%$ & $36.9 \%$ \\
\hline
\end{tabular}


Para os dados registados determinou-se o índice de Tiffeneau (IT).

F.L.C. Lundgren et al. (2006) referem que a utilização do VEM $_{6}$ como substituto da CVF na triagem do diagnóstico da Doença Pulmonar Obstrutiva Crónica, DPOC, parece ser um bom indicador, quando se comparam os valores obtidos por VEM 1 /CVF e VEM 1 /VEM . $_{\text {. }}$.

Os autores que utilizam o volume expiratório máximo ou forçado nos primeiros 6 segundos, VEM 6 ou VEF VE$_{6}$, como substituto da CVF não justificam a opção, apenas referem os resultados como interessantes e causando menos esforço por parte do paciente, pois o exame é obtido num intervalo de tempo menor, e portanto menos invasivo.

Neste trabalho, com base na colecção de dados registada foi possível conhecer a importância de cada VEM na triagem dos distúrbios ventilatórios, e investigar qual seria o melhor VEM $M_{i}$ na substituição da CVF para gerar um indicador para a triagem do diagnóstico da DPOC.

A fig. 2 mostra para homens e mulheres a relação de $V E M_{1} / V E M_{i}$. Em cada gráfico é mostrada a linha teórica de declive unitário para valores de IT. Os círculos de cor azuis representam o indicador $\mathrm{VEM}_{1} / \mathrm{VEM}_{6}$ de modo a ser possível comparar os dados $\operatorname{VEM}_{1} / \mathrm{VEM}_{\mathrm{j}}$ com j de $2 \mathrm{~s}$ a $5 \mathrm{~s}$ e com outras cores.

Aobservação dos gráficos da fig. 2 mostra inequivocamente que devem ser valorizados os indicadores $V E M_{1} / V E M_{4}$ e VEM $1 / V E M_{5}$ em face de VEM $/ V_{1} M_{6}$. No entanto e de acordo com a Tabela 3 cerca de $92 \%$ dos pacientes atinge no máximo um tempo de exame de $4 \mathrm{~s}$, o que valida a importância do uso do indicador $V_{E M} / V_{1} M_{4}$ em face dos indicadores VEM $/ \mathrm{VEM}_{6}$ e VEM 1 /CVF.

A fig. 3 mostra, para homens e mulheres, a correlação entre cada VEM $/ \mathrm{VEM}_{\mathrm{j}}$ com $\mathrm{j}$ de $2 \mathrm{~s}$ a 5 s e o valor de VEM $/$ $V_{E M_{6}}$. Em cada gráfico é mostrada a linha teórica de declive unitário para valores de $\mathrm{VEM}_{1} / \mathrm{VEM}_{6}$. Os círculos de cor cinza representam o indicador $\operatorname{VEM}_{1} / \mathrm{VEM}_{\mathrm{j}}$ de modo a ser possível comparar os dados VEM $1 / V E M_{6}$. Mais uma vez, há uma excelente correlação entre $V E M_{1} / V E M_{4}$ e VEM $M_{1} / V M_{6}$.

A fig. 4 mostra a colecção de dados independentemente do sexo, ou seja a totalidade dos exames em número de 84. Para VEM $/ \mathrm{VEM}_{4}$ e VEM $/ \mathrm{VEM}_{5}$ verifica-se uma excelente concordância com IT, assim como entre VEM / $\mathrm{VEM}_{4}$ e VEM $/ \mathrm{VEM}_{6}$. Os indicadores $\mathrm{MEF}_{50 \%} / 0.5 \mathrm{CVF}$ e IT mostram a existência de um coeficiente de correlação de Pearson de 0.9566 .

Os dois últimos gráficos da fig. 4 mostram que o indicador IT e $\mathrm{MEF}_{50 \%}$ dependem da idade do paciente. A linha a cheio representa a tendência dos valores previstos quando se aplica o método dos desvios mínimos quadráticos, os círculos de cor a verde a previsão através da aplicação de modelos teóricos e os círculos de cor azul a amostra estudada. A observação destes dois gráficos mostra que há uma grande dispersão dos pontos experimentais em face da linha de tendência o que suscita que há casos em que distúrbios de ventilação de pacientes devem ser valorizados.

Uma análise de pormenor foi considerada valorizando a existência de DPOC. A fig. 5 apresenta diferentes gráficos para interpretar a triagem de diagnóstico de DPOC, usando o IT e o $\mathrm{MEF}_{50 \%} / 0.5 \mathrm{CVF}$ como função da idade do paciente. Os círculos ocos representam os valores previstos, os triângulos ocos os valores normais, a linha a cheio a tendência usando o método dos desvios médios quadráticos, os círculos de cor vermelha situação de obstrução, os círculos de cor azul de restrição e os círculos de cor a verde o misto de obstrução e restrição.

A observação dos gráficos da fig. 5 mostra, como era esperado, que para a restrição (DVR) os valores de IT não mostram patologia, mas o $\mathrm{MEF}_{50 \%}$ (fluxo expiratório a $50 \%$ da capacidade vital) mostra um desvio absoluto significativo, o que permite interpretar que há distúrbios de ventilação por restrição. Para a obstrução (DVO) os valores estão de acordo com o esperado, assim como para a situação mista de obstrução e restrição.

A fig. 6 mostra excelente concordância entre os indicadores $\mathrm{MEF}_{50 \%} / 0.5 \mathrm{CVF}$ e IT. No gráfico do lado esquerdo são mostrados também os casos normais a círculo a negrito. Os círculos a cores indicam como o indicado na fig. 5, a valorização de diagnóstico de DPOC (casos de DVO e DVR).

A literatura de especialidade não apresenta estudos que valorizem um parâmetro adimensional que deve caracterizar o tipo de escoamento, denominado número de Reynolds (Re). Este mede a razão entre as forças de inércia e as forças viscosas (B.S. Massey, 2002). Foi nesta perspectiva que se valorizou um novo indicador que considerasse o fluxo expiratório a $50 \%$ da capacidade vital expulso numa expiração forçada, através do Re. A velocidade média do fluido [calculada a partir do caudal volumétrico e do diâmetro do bucal do tubo (D)] e as propriedades físicas do fluido (massa volúmica $\rho$ e viscosidade dinâmica $\mu)$ determinam o $\operatorname{Re}(=\rho v D / \mu)$. No caso do aparelho usado neste trabalho, o diâmetro interior de passagem do fluido, na zona de controlo, é de $21 \mathrm{~mm}$.

A fig. 7 mostra que o número de Reynolds depende da idade do paciente, como seria esperado. Os círculos ocos representam os valores previstos, a linha a cheio a tendência quando se aplica aos valores previstos o método dos desvios quadráticos médios, os círculos de cor vermelha situação de obstrução, os círculos de cor azul de restrição e os círculos de cor a verde o misto de obstrução. 


\section{territorium 24}

A observação do gráfico da fig. 7 mostra inequivocamente que quando os valores obtidos estão localizados abaixo da linha de tendência devem ser valorizados em termos de triagem de diagnóstico de distúrbios ventilatórios. $\mathrm{Na}$ prática, há estrangulamento ou situação de quebra de elasticidade pulmonar para a expulsão do ar.

\section{Homens}
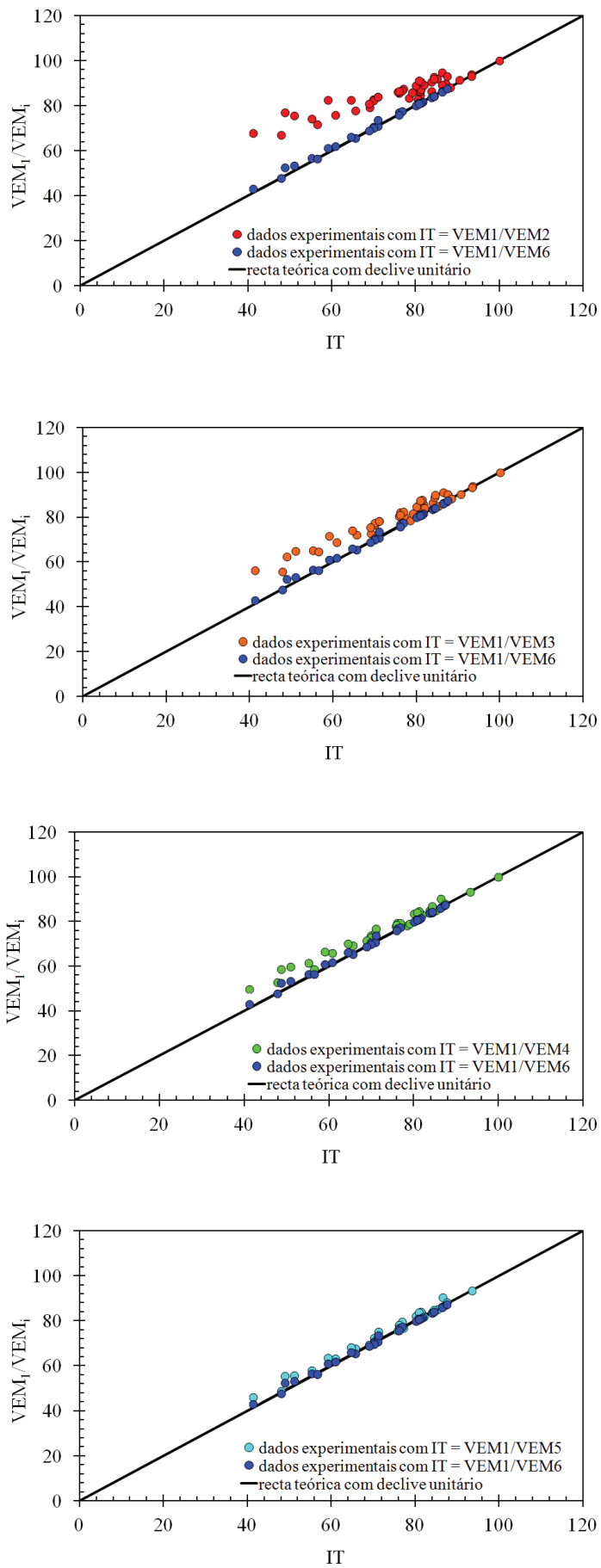

A interpretação física do número de Reynolds mostra que os valores muito abaixo do Re indicados pela linha de tendência devem ser valorizados para a triagem de DPOC, patologia DVO ou DVR, ou seja, os valores mostram uma queda da força de inércia em face da força viscosa, o que indicia patologia. Nestes termos, o
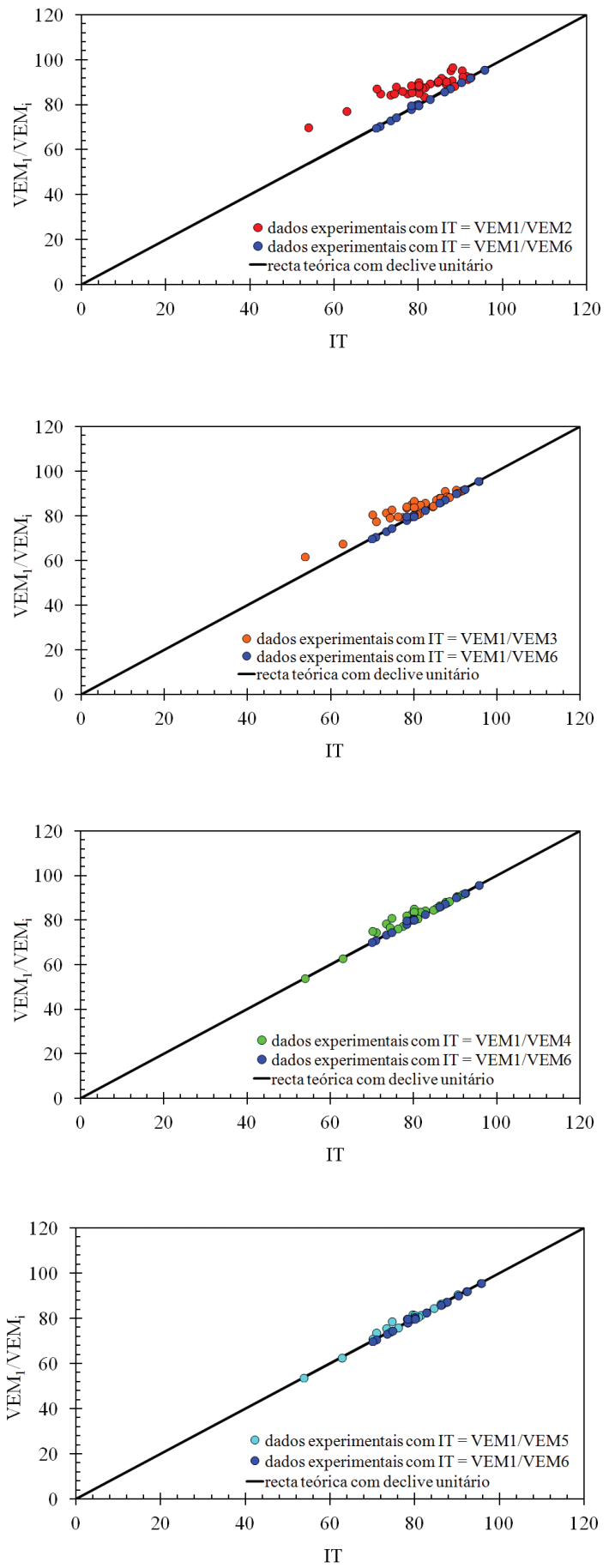

Fig. 2 - Dados da amostra analisada em termos de VEM $/$ VEM e IT (homens e mulheres).

Fig. 2 - Sample data analyzed in terms of $\mathrm{FEV}_{1} / \mathrm{FEV}_{i}$ and $\mathrm{TI}$ (men and women). 
número de Reynolds parece ser um parâmetro que deve ser valorizado na triagem de diagnóstico de distúrbios ventilatórios. De facto, todos os casos de DVO ou DVR ou DVO+R mostram a sua localização muito abaixo da linha de tendência, o que valida o que anteriormente foi referido.

\section{Homens}
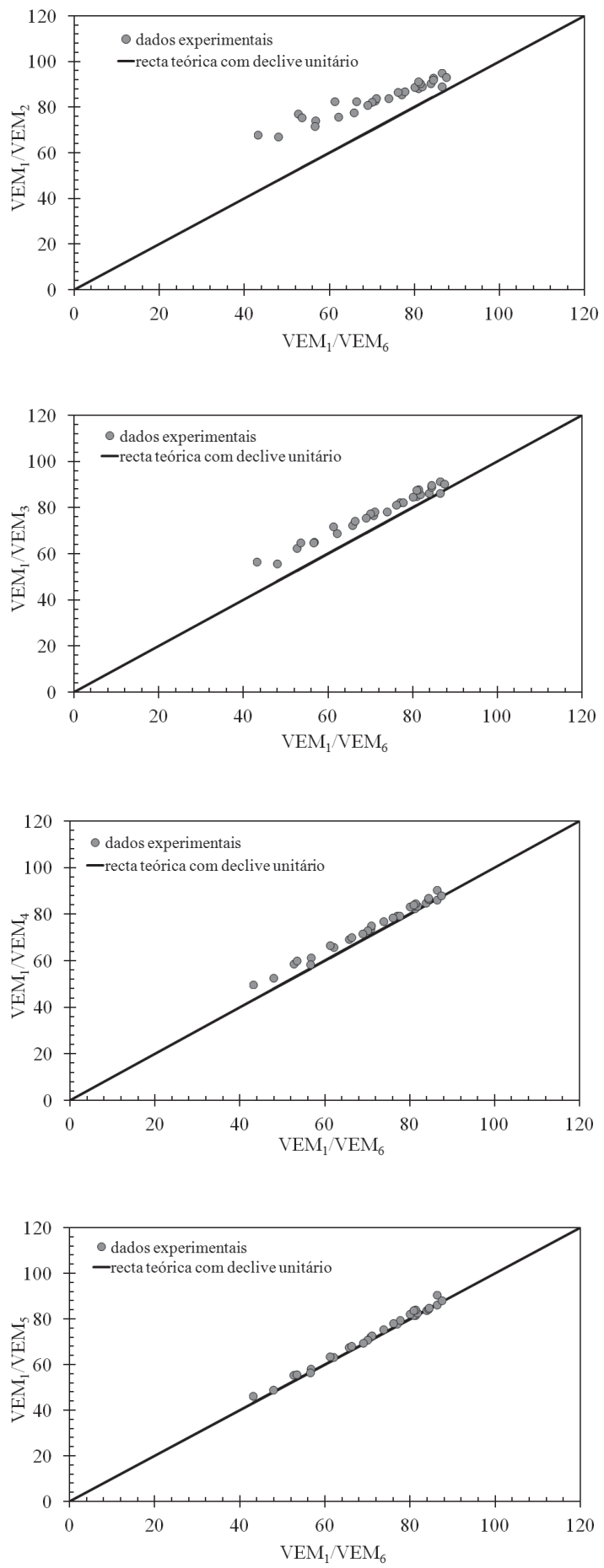

\section{Conclusão}

Este trabalho mostra como o indicador $V_{1} M_{1} / V M_{6}$, desenvolvido por vários autores, deve ser valorizado face ao indicador VEM $/$ CVF. No entanto este trabalho veio revelar que se for utilizado o indicador $\mathrm{VEM}_{1} /$

\section{Mulheres}
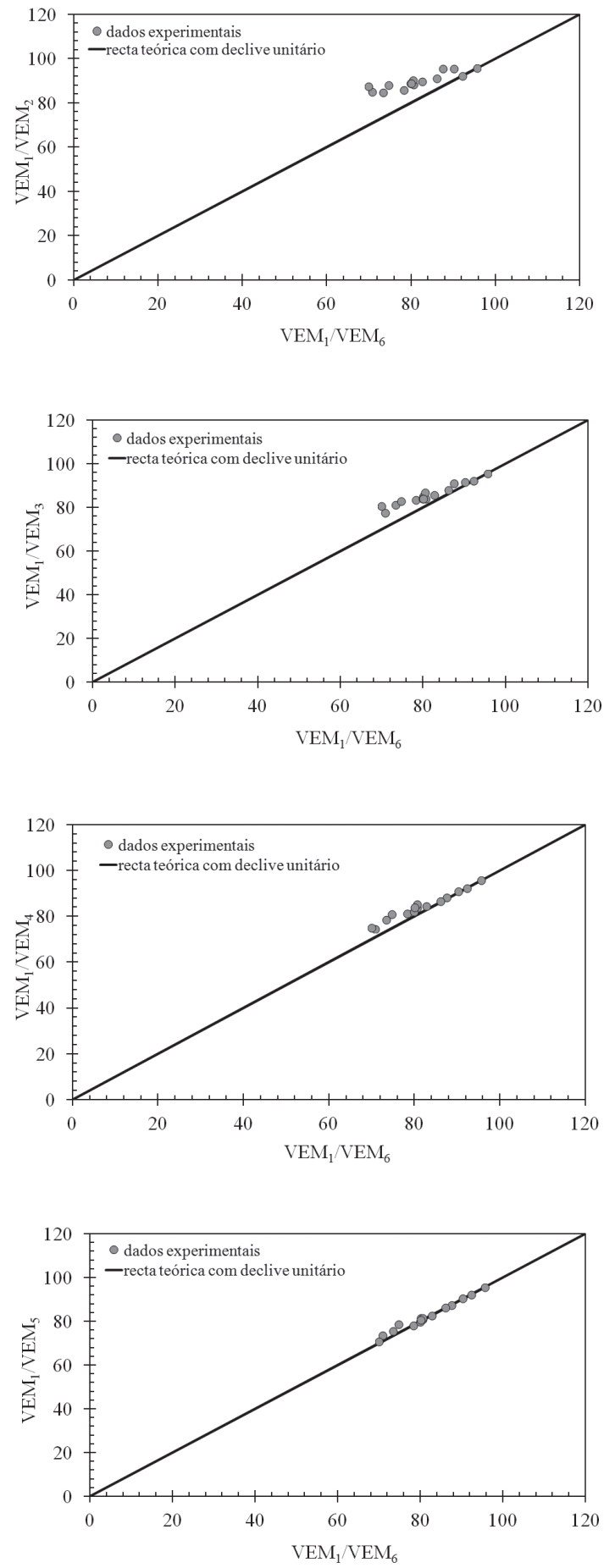

Fig. 3 - Dados da amostra analisada em termos de $V E M_{1} / V E M i$ e $V E M_{1} / V_{\mathrm{MG}_{6}}$ (homens e mulheres).

Fig. 3 - Sample data analyzed in terms of FEV $/$ FEV $_{i}$ and FEV ${ }_{1} /$ FEV $_{6}$ (men and women). 
$\mathrm{VEM}_{4}$, menos invasivo para o paciente por usar apenas 4 segundos, é possível proceder também à triagem de diagnóstico de distúrbios ventilatórios. Na prática, os registos mostraram que cerca de $92 \%$ dos resultados obtidos para os pacientes confirmaram um exame com a duração até $4 \mathrm{~s}$.

Os resultados obtidos permitiram construir diversos gráficos para os sexos masculino e feminino e para casos considerados de normais, obstrutivos e restritivos. Foi possível correlacionar os dados determinados por $\mathrm{MEF}_{50 \%} / 0,5 \mathrm{CVF}$ e VEM $/$ CVF. O estudo mostrou que a substituição de CVF por VEM $\mathrm{M}_{4}$ é uma estratégia que deve ser valorizada para a triagem de diagnóstico de DVO e DVR.

Os resultados obtidos mostraram que a triagem de diagnóstico de distúrbios ventilatórios deve valorizar não só o valor do índice de Tiffeneau mas também o valor de $\mathrm{MEF}_{50 \%} / 0,5 \mathrm{CVF}$, pois só a valorização dos dois em simultâneo permite conhecer os casos de patologia DVO e DVR.

Um novo indicador para o diagnóstico da DPOC foi investigado e testado. A interpretação física do número de Reynolds mostra que os valores muito abaixo do Re indicados pela linha de tendência devem ser valorizados para a triagem de DPOC, patologia DVO ou DVR, ou seja, os valores mostram uma queda da força de inércia face à força viscosa.

Os resultados obtidos mostraram que o número de Reynolds função da idade do paciente deve ser valorizado na triagem de diagnóstico de distúrbios ventilatórios.

Em trabalhos futuros sugere-se que sejam valorizados parâmetros termohigrométricos (M.P. Strauss et al.,
Homens
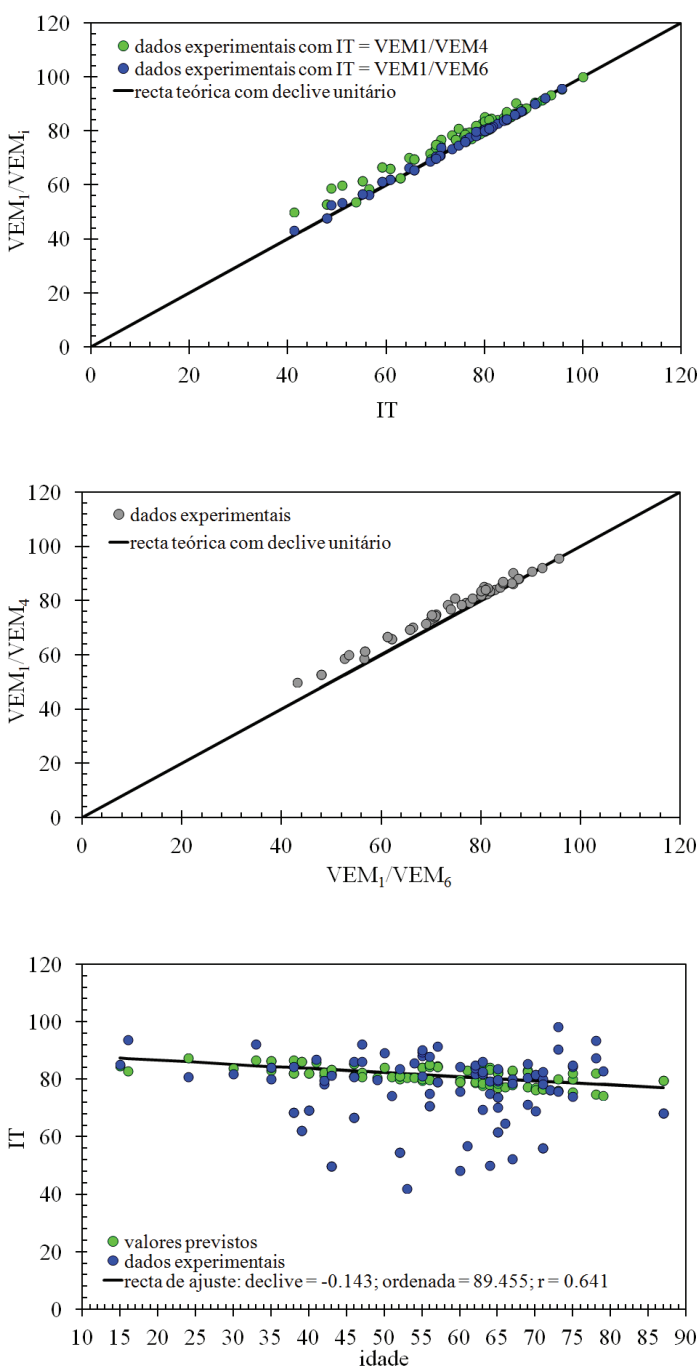

Mulheres
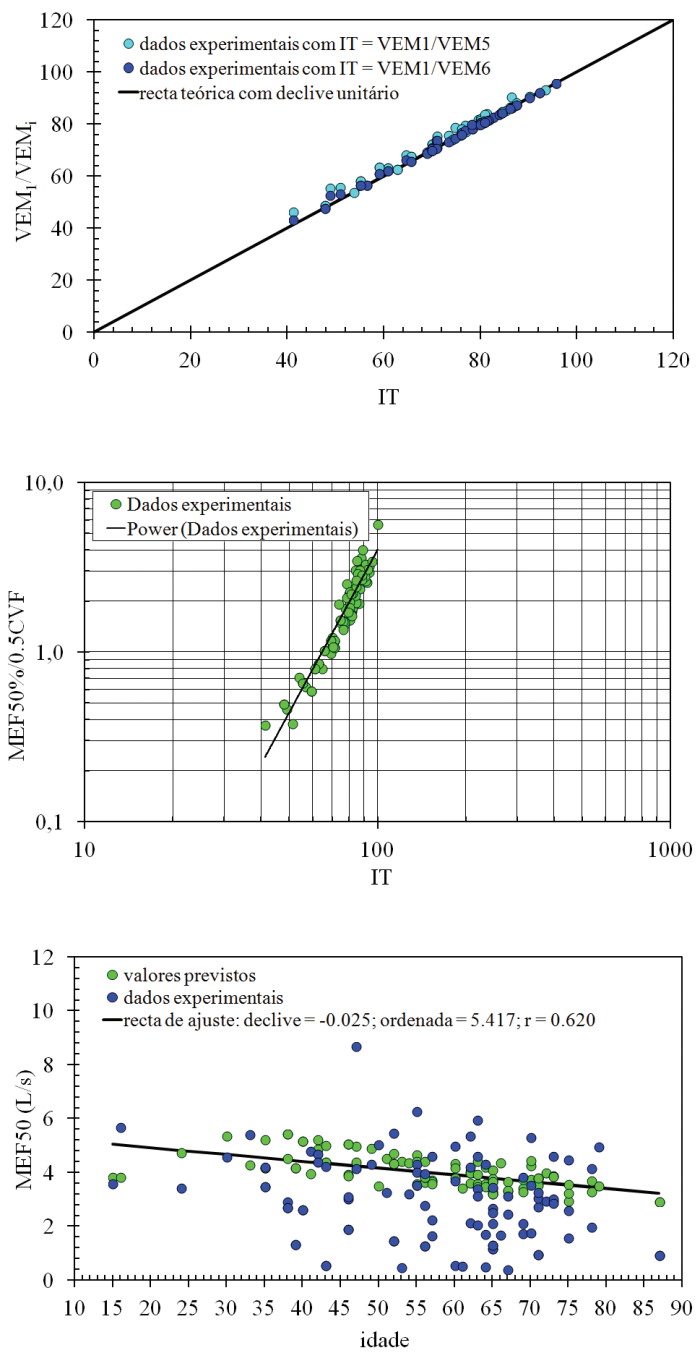

Fig. 4 - Dados de toda a amostra em diferentes analises.

Fig. 4 - All the sample data in different analysis. 

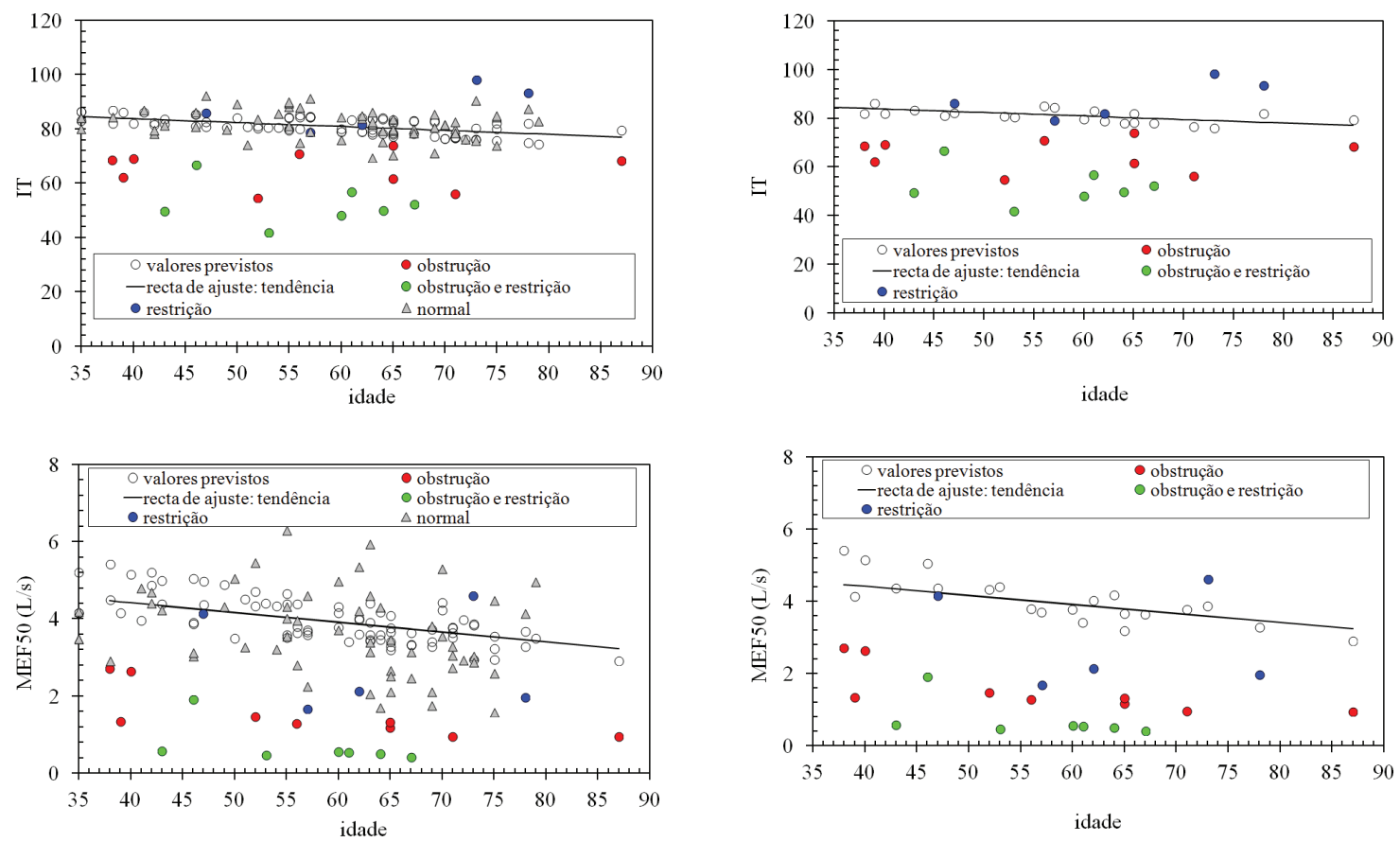

Fig. 5 - Diagnóstico de DPOC (indicador IT e MEF50\% função da idade).

Fig. 5 - COPD diagnosis (TI indicator and MEF50\% depending on age).
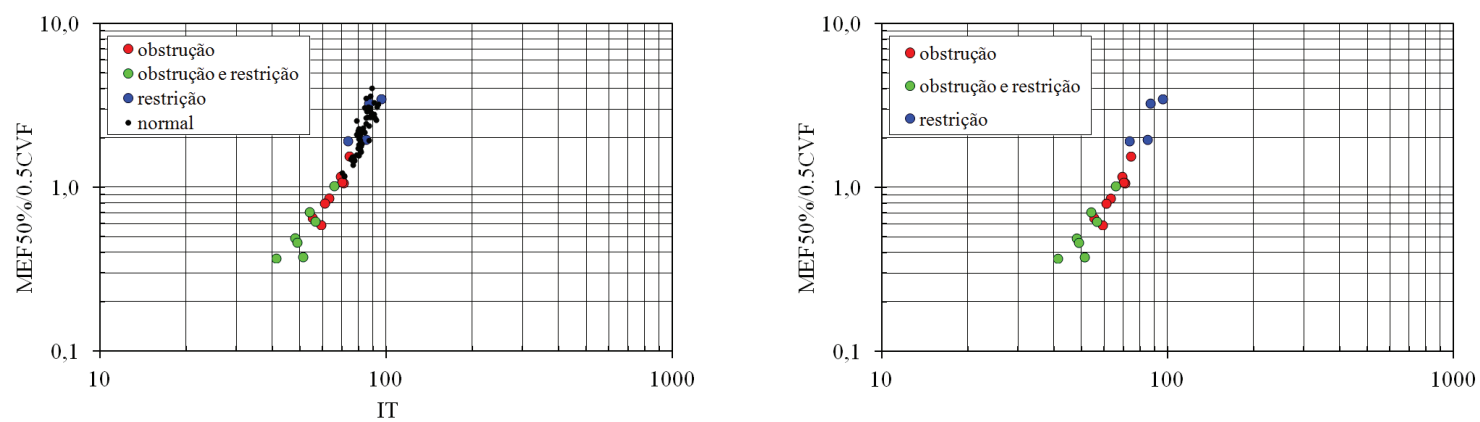

Fig. 6 - Diagnóstico de DPOC (correlação entre indicador o IT e o MEF50\%/0.5CVF).

Fig. 6 - COPD diagnosis (correlation between TI indicator and MEF50\%/0.5FVC).

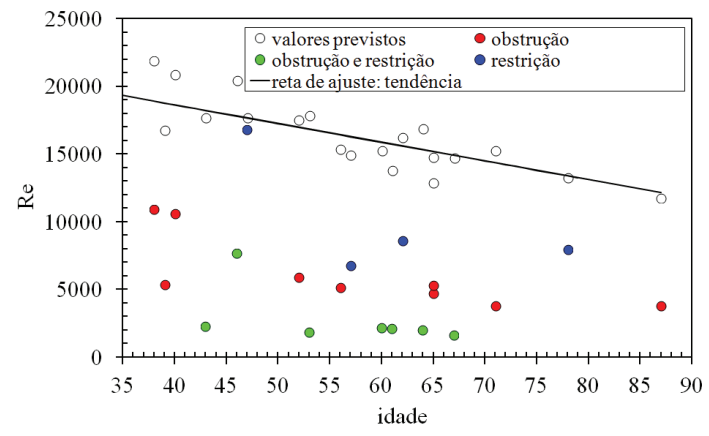

Fig. 7 - Número de Reynolds condiciona o tipo de escoamento com a idade

Fig. 7 - The Reynolds number determines the type of flow with age.
1978; O. Heikki et al., 1966), assim como o registo da pressão absoluta do ar no bocal do tubo como parâmetro condicionante de triagem de diagnóstico a partir do número de Reynolds.

\section{Agradecimentos}

Um agradecimento é expresso à Doutora Alcina Saraiva médica especialista dos serviços de pneumologia do Hospital da região centro de Portugal que colaborou na realização deste trabalho contribuindo com toda a sua sabedoria e experiência. As técnicas Elisabete e Lucinda pela sua disponibilidade e excelente profissionalismo no registo da colecção de dados. 


\section{Referências bibliográficas}

Aaron, S. D., Dales, R. E. e Cardinal, P. (1999). How accurate is spirometry at predicting restrictive pulmonary impairment? Chest, 115:869-873.

Almeida, C., Talaia, Mário e Saraiva, A. (2012). Espirometria como teste de Diagnóstico de robustez Física - Estudo de caso de uma população. Livro de res VII Encontro Nacional de Riscos e I Fórum sobre Riscos e Segurança ISCIA. Editor FEDRAVE / Mare Liberum. ISCIA - Aveiro. 19-20 de Abril, 56.

Arne, M., Lisspers, K., Stallberg, B., Boman, G. Hedenstrom, H., Janson, C. e Emtner, M. (2009). How often is diagnosis of CPOD confirmed with spirometry? Respiratory Medicine, 104:550-556.

CAREFUSION CORPORATION (2012). MasterScreen Body, JAEGER, Cardinal Health, SensorMedics, Hoechberg, Germany.

Chowienczyk, P. J. e Lawson, C. P. (1982). Pocket sized device for measuring forced expiratory volume in are one second and forced vital capacity. British Medical Journal, 285(7):15-17.

Clausen, J. L. (1989). Clinical interpretation of pulmonary function tests. Respiratory Care, 146:1779-1781.

Cohen, L., Manion, L. e Morrison, K. (2010). Research Methods in Education (6a edição). New York: Routledge.

Denzin, N.K., e Lincoln, Y. S. (2005). Handbook of Qualitativ Research. Thousand Oaks: Sage.

Eaton, T., Withy, S. e Garrett, J. E. (1999a). Spirometry in primary care practice. The importance of quality assurance and the impact of spirometry workshops. Chest, 116:416-423.

Eaton, T., Withy, S., Garrett, J. E., Mercer, J., Whitlock, R. M. L. e Rea, H. (1999b). Spirometry in Primary Care Practice: The importance of Quality assurance and the spirometry. Chest, 116:416-423.

Enright, P. L., Connet, J. E. e Bailey, W.C. (2002). The $\mathrm{FEF}_{1} / \mathrm{FEF}_{6}$ predicts lung function decline in adult smokers. Respiratory Medicine, 96:444-449.

Fergunson, G., Enright, P. L., Buist, A. S. e Higgins, M. (2000). Office spirometry for lung health assessment in adults. A consensus statement from the National Lung Health Education Program. Chest, 117:1146-1161.

Gleeson, S., Mitchell, B., Pasquarella, C., Reardon, E., Falsone, J. e Berman, L. (2006). Comparison of $\mathrm{FEF}_{6}$ and FVC for detection of airway obstruction in a community hospital pulmonary function laboratory. Respiratory Medicine, 100(8):1397-401.

Green, M., Mead, J. e Turner, J. M. (1974). Variability of maximum expiratoty flow-volume curves. J Appl Physiol., 37(1):67-74.
GOLD (2006). Global Strategy for the Diagnosis, Management and Prevention of Chronic Obstructive Pulmonary Disease. Global Initiative for chronic obstructive lung disease. [GOLD Website].

Heikki, O., Koskela, K., Koskela, A., Hannu, O. e Tukiainen, O. (1996). Bronchoconstriction due to Cold Mechanisms Airway Effects and Cutaneous Reflex Weather in COPD. Chest, 110:632-636.

Huot, R. (2002). Métodos Quantitativos para Ciências Humanas. Lisboa: Instituto Piaget, Col. Epistemologia e Sociedade.

Hyatt, R. E., Scanlon, P. D. e Nakamura, M. (1997). Interpretation of pulmonary function tests. A practical guide. Lippincontt-Raven. 27-39.

Jakob, M. (2009). Euromédice. BÖrm Bruckmeier Verlag. AstraZeneca Respiratória.

Joseph, D., Cherniack, R., Paull, K. e Gelfand, E. W. (2004). Is Forced Expiratory Volume in one second the Best measure of severity in childhood asthma? Am J Respir Crit Care Med, 169:784-786.

Knudson, R. J., Lebowitz, M. D., Holberg, C. J. e Burrows, B. (1983). Changes in the normal maximal expiratory flow-volume curve with growth and aging. Am Rev Respir Dis, 127(6):725-734.

Knudson, R. J., Slatin, R. C., Lebowitz, M. D. e Burrows, B. (1976). The maximal expiratory flow-volume curves. Normal standards variability and effect of age. Am Rev Respir Dis, 113:587-600.

Lopez, A. D., Mathers, C. D., Ezzati, M., Jamison, D. T. e Murray, C.J. (2001). Global and regional burden of disease and risk factors: systematic analysis of population health data. Lancet, 367(9524):1747-1757.

Lundgren, F. L. C., Cabral, M. M., Clímaco, D. C. S., Macedo, L. G., Coelho, M. A. L. e Dias, A. L. P. L. A. (2006). Determinação da eficiência do $\operatorname{VEF}_{6}$ como substituto da CVF na triagem diagnóstica da doença pulmonar obstrutiva crónica através da comparação entre as relações $\mathrm{VEF}_{1} / \mathrm{CVF}$ e $\mathrm{VEF}_{1} / \mathrm{VEF}_{6}$. Journal Brasilian Pneumology, 33(2):148-151.

Massey, B.S. (2002). Mecânica dos Fluidos. Fundação Calouste Gulbenkian, Lisboa.

Miller, M. R., Hankinson, J., Brusasco, V., Burgos, F., Casaburi, R., Coates, A., Crapo, R., Enright, C.P.M., Van der Grinten, P., Gustafsson, R., Jensen, R., Johnson, D.C., Maclntyre, N., McRay, R., Navajas, O.F., Pedersen, O.F., Pellegrino, R., Viegi, G. e Wanger, J. (2005). Standardisation of spirometry. European Respiratory Journal, 26:319-338.

Murray, C. J. e Lopez, A. D. (1997). Alternative projections of mortality and disability by cause 
1990-2020: Global Burden of Disease Study. Lancet, 349(9064):1498-1504.

O’Donnell, D. E., Lam, M. e Webb, K. A. (1999). Spirometric correlates of improvement in exercise performance after anticholinergic therapy in chronic obstructive pulmonary disease. Am J Respir Crit Care Med, 160:542-549.

Oga, T., Nishimura, K., Tsukino, M., Sato, S. e Hajiro, T. (2003). Analysis of the factors related to mortality in chronic obstructive pulmonary disease. Am J Respir Crit Care Med., 167(4):544-549.

Papaioannou, A. I., Loukides, S., Gourgoulianis, K.I. e Kostikas, K. (2009). Global assessment of the COPD patient: Time to look beyond $\mathrm{FEV}_{1}$ ? Respiratory Medicine, 103:650-660.

Pardal, L. e Lopes, E.S. (2011). Métodos e Técnicas de Investigação Social. Porto: Areal Editores.

Pereira, C.A.C. (2002). Espirometria. J Pneumol, 28(3):1-82.

Pereira, C.A.S., Sato, T. e Rodrigues, S.C. (2007). Novos valores de referência para espirometria forçada em brasileiros adultos de raça branca. J Bras Pneumol, 33(4):397-406.

Quanjer, P. H., Tammeling, G. J., Cotes, J. E., Pedersen, R., Peslin, R. e Yearnault, J.C. (1993). Lung volumes and forced ventilator flows: report of working party, standardization of lung function tests. European Community for steel and coal - official statement of the European Respiratory Society. European Respiratory Journal, 6(16): 5-40.

Rodrigues, M.T., Fiterman-Molinari, D., Barreto, S.S.M. e Fiterman, J. (2010). O papel da razão $\mathrm{FEF}_{50 \%} / 0,5 \mathrm{CVF}$ no diagnóstico dos distúrbios ventilatórios obstrutivos. Journal Brasilian Pneumology, 36 (1): 44-50.

Silva, L. C. C., Rubin, A. S., Silva, L. M. C. e Fernandes, J.C. (2005). Espirometria na prática médica. Revista AMRIGS, 49(3):183-194.
Spahn, J. D., Cherniack, R., Paull, K. e Gelfand, E. W. (2004). Is Forced Expiratory Volume in One Second the Best Measure of Severity in Childhood Asthma? Am. J. Respir. Crit. Care Med., 169:784-786.

Strauss, R. H., McFadden, E. R., Ingran, R. M. e Chandler, E. (1978). Influence of heat and humidity on the airway obstruction induced by exercise in asthma. Journal of Clinical Investigation, 61(2):433-440.

Swanney, M. P., Beckert, L. E., Frampton, C. M., Wallace, L. A., Jensen, R. L. e Crapo, R. O. (2004). Validity of the American Thoracic Society and other spirometric algorithms using FVC and forced expiratory volume at $6 \mathrm{~s}$ for predicting a reduced total lung capacity. Chest, 126(6):1861-1866.

Swanney, M. P., Jensen, R. L., Crichton, D. A., Beckert, L. E., Cardno, L. A. e Crapo, R. O. (2000). FEV 6 is an acceptable surrogate for FVC in the spirometric diagnosis of airway obstruction and restriction. Am J Repir Crit Care Med, 162(3Pt1):917-919.

Terra Filho, J. (1998). Avaliação Laboratorial da Função Pulmonar. Medicina, 31:191-207.

Vandevoorde, J., Verbanck, S., Schuermans, D., Kartounian, J. e Vinken, W. (2005). $\mathrm{FEF}_{1} / \mathrm{FEF}_{6}$ and $\mathrm{FEF}_{6}$ as an alternative for $\mathrm{FEF}_{1} / \mathrm{FVC}$ and $\mathrm{FVC}$ in the spirometric detection of airway obstruction and restriction. Chest, 127(5):1560-1564.

White, P. (2003). Spirometry and peak expiratory flow in the primary care management of COPD. Primary Care Journal, 13:5-8.

Wise, R. A. e Tashkin, D.P. (2007). Preventing chronic obstructive pulmonary disease: What is known and what needs to be done to make a difference to the patient? The American Journal of Medicine, 120(8A), S14-S22. 